Supplement of Biogeosciences, 12, 4751-4764, 2015

http://www.biogeosciences.net/12/4751/2015/

doi:10.5194/bg-12-4751-2015-supplement

(C) Author(s) 2015. CC Attribution 3.0 License.

(c) (i)

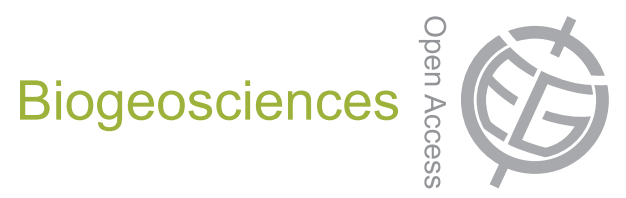

Supplement of

\title{
Nitrogen fixation and the diazotroph community in the temperate coastal region of the northwestern North Pacific
}

\section{T. Shiozaki et al.}

Correspondence to: T. Shiozaki (shiozaki@aori.u-tokyo.ac.jp)

The copyright of individual parts of the supplement might differ from the CC-BY 3.0 licence. 


\section{Introduction}

The supplementary material for this manuscript consists of one table and five figures. The figure captions including methodology are given below.

Fig. S1. Sampling locations for each cruise in the northwestern North Pacific Ocean. Background contours and vectors denote the sea surface temperature (SST) and geostrophic current field during the cruise period. The SST dataset was the daily MODIS level-3 SST with 4-km resolution, and was obtained from the NASA Goddard Space Flight Center (http://oceancolor.gsfc.nasa.gov/). Weekly geostrophic current field data with $1 / 3^{\circ}$ for approximately the same period as the cruise were obtained from the AVISO data server (ftp.aviso.oceanobs.com). The gray line during the KK-13-6_Sep cruise indicates the track of Typhoon Man-yi. The flow volume of the Tsushima Water Current is recognized to be largest in summer and makes an anticyclonic eddy on the Pacific side, and is smallest in winter when it does not form the eddy structure (Conlon, 1982; Nishida et al., 2003). During the KT-12-20_Aug, KT-12-27_Oct, KK-13-1_Jun, and KK-13-6_Sep cruises, the anticyclonic eddy was observed on the Pacific side, and the water flowed southward along the coast. The Tsugaru Warm water during the KT-13-2_Jan cruise immediately flowed along the coast after it passed the Tsugaru Strait. Although the southward current subsided at the observation lines during the KT-13-2_Jan cruise, all the water at the surface belonged to the Tsushima Water Current. During the KS-14-2_Mar cruise, the flow from the Tsugaru Strait was weak, and water with a temperature less than $3^{\circ} \mathrm{C}$ spread to the south of the OT transect line, suggesting that the OT transect line was influenced by the Oyashio water. During the KK-13-6_Sep cruise, a low SST belt was observed 
from south to north on the Pacific side. This belt almost corresponded with the typhoon track, and thus was caused by the passage of the typhoon.

Fig. S2. Surface spatial distributions of ( $a$ and $b)$ nitrate and (c and d) ammonium concentration $[\mu \mathrm{M}]$ and (e and $\mathrm{f}$ ) the nitrogen fixation rate $\left[\mathrm{nmol} \mathrm{N} \mathrm{L} \mathrm{L}^{-1} \mathrm{~d}^{-1}\right]$ along the OT and ON transect lines for each cruise.

Fig. S3. Maximum likelihood phylogenetic trees of nifH amino acid sequences of Proteobacteria in Cluster I. The recovered sequences in this study are in boldface type. The boldface numbers in parentheses are the number of retrieved clones for each cruise and station. Bootstrap values $(>50 \%)$ are indicated at branch points. Sequences with an asterisk have $>97 \%$ similarity at the amino acid level with terrestrial diazotrophs, and with sequences derived from soil, mudflats, and lakes.

Fig. S4. Maximum likelihood phylogenetic trees of nifH amino acid sequences of Cyanobacteria in Cluster I. The recovered sequences in this study are in boldface type. The boldface numbers in parentheses are the number of retrieved clones for each cruise and station. Bootstrap values $(>50 \%)$ are indicated at branch points. Sequences with an asterisk have $>97 \%$ similarity at the amino acid level with terrestrial diazotrophs.

Fig. S5. Maximum likelihood phylogenetic trees of nifH amino acid sequences in Cluster III. The recovered sequences in this study are in boldface type. Boldface numbers in parentheses are the number of retrieved clones for each cruise and station. Bootstrap values $(>50 \%)$ are indicated at branch points. Sequences with an asterisk 
have $>97 \%$ similarity at the amino acid level with sequences derived from mudflats.

Fig. S6. Surface spatial distributions of (a and b) Trichodesmium, (c and d) UYCN-A, (e and f) UCYN-B, and (g and h) $\gamma$-24774A11 $\left[\log _{10}\left((\right.\right.$ copy +1$\left.\left.) L^{-1}\right)\right]$ along the OT and ON transect lines for each cruise.

Fig. S7. Vertical profiles of salinity along the OT transect line during the KK-13-6_Sep cruise.

Fig. S8. Vertical profiles of the oxygen concentration $\left[\mathrm{ml} \mathrm{L}^{-1}\right]$ for each cruise.

\section{REFERENCES}

Conlon, D. M.: On the outflow modes of the Tsugaru Warm Current, La mer, 20, 60-64, 1982.

Nishida, Y., Kanomata, I., Tanaka, I., Sato, S., Takahashi, S., and Matsubara, H.: Seasonal and interannual variations of the volume transport through the Tsugaru Strait, Oceanogr. Japan, 12(5), 487-499, 2003. 
Table S1 Water properties and nitrogen fixation used for Pearson's correlation analysis

\begin{tabular}{|c|c|c|c|c|c|c|c|c|c|c|}
\hline Cruise & Station & Latitude & Longitude & Depth & Temperature $\left[{ }^{\circ} \mathrm{C}\right]$ & Nitrate $[\mu \mathrm{M}]$ & Ammonium $[\mu \mathrm{M}]$ & Phosphate $[\mu \mathrm{M}]$ & N/P ratio & $\mathrm{N}_{2}$ fixation $\left[\mathrm{nmolN} \mathrm{L}^{-1} \mathrm{~d}^{-1}\right]$ \\
\hline KT-12-20_Aug & OT1 & 39.34295 & 141.9351 & 0 & 21.9 & 0.05 & 0.22 & 0.20 & 0.25 & 2.84 \\
\hline KT-12-20_Aug & OT4 & 39.33318 & 142.1663 & 0 & 21.7 & $<0.02$ & 0.03 & $<0.01$ & 5.37 & 10.8 \\
\hline KT-12-20_Aug & OT4 & 39.33318 & 142.1663 & 11 & 19.5 & $<0.02$ & $<0.03$ & $<0.01$ & 5.00 & 12.0 \\
\hline KT-12-20_Aug & OT4 & 39.33318 & 142.1663 & 34 & 15.8 & $<0.02$ & $<0.03$ & $<0.01$ & 5.00 & 1.20 \\
\hline KT-12-20_Aug & OT4 & 39.33318 & 142.1663 & 63 & 13.2 & 2.80 & $<0.03$ & 0.36 & 9.15 & n.d. \\
\hline KT-12-20_Aug & OT5 & 39.33357 & 142.4981 & 0 & 23.9 & $<0.02$ & $<0.03$ & $<0.01$ & 5.00 & 8.50 \\
\hline KT-12-20_Aug & OT6 & 39.3318 & 142.8352 & 0 & 24.3 & $<0.02$ & $<0.03$ & $<0.01$ & 5.00 & 0.49 \\
\hline KT-12-20_Aug & ON1 & 38.42083 & 141.4858 & 0 & 22.4 & $<0.02$ & 0.05 & $<0.01$ & 7.39 & 2.82 \\
\hline KT-12-20_Aug & ON4 & 38.41697 & 141.7507 & 0 & 20.6 & $<0.02$ & $<0.03$ & 0.08 & 0.67 & 3.34 \\
\hline KT-12-20_Aug & ON5 & 38.41721 & 142 & 0 & 22.3 & $<0.02$ & 0.02 & 0.03 & 1.42 & 5.02 \\
\hline KT-12-20_Aug & ON5 & 38.41721 & 142 & 9 & 21.8 & $<0.02$ & $<0.03$ & $<0.01$ & 5.00 & 3.79 \\
\hline KT-12-20_Aug & ON5 & 38.41721 & 142 & 34 & 11.9 & 0.85 & $<0.03$ & 0.15 & 6.10 & n.d. \\
\hline KT-12-20_Aug & ON5 & 38.41721 & 142 & 62 & 10.1 & 8.05 & $<0.03$ & 0.69 & 11.7 & n.d. \\
\hline KT-12-20_Aug & ON6 & 38.41782 & 142.3326 & 0 & 22.5 & $<0.02$ & 0.11 & 0.05 & 2.63 & 7.95 \\
\hline KT-12-20_Aug & ON7 & 38.41569 & 142.6659 & 0 & 21.0 & $<0.02$ & $<0.03$ & 0.13 & 0.39 & n.d. \\
\hline KT-12-27_Oct & OT1 & 39.34597 & 141.954 & 0 & 20.3 & 0.11 & 0.05 & 0.10 & 2.42 & n.d. \\
\hline KT-12-27_Oct & OT4 & 39.33318 & 142.1663 & 0 & 20.0 & $<0.04$ & 0.08 & 0.08 & 1.37 & 0.45 \\
\hline KT-12-27_Oct & OT4 & 39.33318 & 142.1663 & 31 & 19.8 & 0.04 & 0.05 & 0.06 & 1.84 & 1.80 \\
\hline KT-12-27_Oct & OT4 & 39.33318 & 142.1663 & 62 & 16.3 & 3.17 & 0.05 & 0.32 & 11.2 & 0.30 \\
\hline KT-12-27_Oct & OT5 & 39.33357 & 142.4981 & 0 & 19.7 & $<0.04$ & 0.08 & 0.07 & 1.70 & n.d. \\
\hline KT-12-27_Oct & OT6 & 39.3318 & 142.8352 & 0 & 19.8 & $<0.04$ & 0.10 & 0.08 & 1.64 & 0.33 \\
\hline KT-12-27_Oct & ON1 & 38.42527 & 141.4883 & 0 & 21.2 & 0.07 & 0.10 & 0.15 & 1.80 & 0.59 \\
\hline KT-12-27_Oct & ON4 & 38.41697 & 141.7507 & 0 & 20.2 & $<0.04$ & 0.06 & 0.07 & 1.49 & 2.26 \\
\hline KT-12-27_Oct & ON5 & 38.41721 & 142 & 0 & 20.8 & $<0.04$ & 0.05 & 0.06 & 1.64 & n.d. \\
\hline KT-12-27_Oct & ON5 & 38.41721 & 142 & 32 & 20.8 & 0.11 & 0.05 & 0.06 & 3.07 & n.d. \\
\hline KT-12-27_Oct & ON5 & 38.41721 & 142 & 65 & 13.3 & 5.77 & 0.06 & 0.50 & 11.7 & n.d. \\
\hline KT-12-27_Oct & ON7 & 38.41569 & 142.6659 & 0 & 20.3 & $<0.04$ & 0.62 & 0.09 & 7.40 & 0.72 \\
\hline KT-13-2_Jan & OT1 & 39.34597 & 141.954 & 0 & 9.8 & 5.22 & 0.18 & 0.47 & 11.9 & n.d. \\
\hline KT-13-2_Jan & OT4 & 39.33318 & 142.1663 & 0 & 9.8 & 6.16 & 0.16 & 0.50 & 12.8 & n.d. \\
\hline KT-13-2_Jan & OT4 & 39.33318 & 142.1663 & 41 & 9.6 & 6.11 & 0.16 & 0.50 & 12.7 & n.d. \\
\hline KT-13-2_Jan & OT4 & 39.33318 & 142.1663 & 76 & 9.4 & 6.30 & 0.16 & 0.52 & 12.6 & n.d. \\
\hline KT-13-2_Jan & OT5 & 39.33357 & 142.4981 & 0 & 9.3 & 6.47 & 0.15 & 0.52 & 12.8 & n.d. \\
\hline KT-13-2_Jan & OT6 & 39.3318 & 142.8352 & 0 & 9.8 & 6.16 & 0.16 & 0.50 & 12.8 & n.d. \\
\hline KT-13-2_Jan & ON1 & 38.42527 & 141.4883 & 0 & 8.8 & 0.75 & 0.35 & 0.27 & 4.50 & n.d. \\
\hline KT-13-2_Jan & ON4 & 38.41697 & 141.7507 & 0 & 9.0 & 6.93 & 0.14 & 0.55 & 13.1 & n.d. \\
\hline KT-13-2_Jan & ON5 & 38.41721 & 142 & 0 & 8.3 & 7.95 & 0.07 & 0.63 & 12.8 & n.d. \\
\hline KT-13-2_Jan & ON5 & 38.41721 & 142 & 62 & 7.3 & 9.84 & 0.07 & 0.80 & 12.4 & n.d. \\
\hline KT-13-2_Jan & ON5 & 38.41721 & 142 & 119 & 6.5 & 12.0 & 0.06 & 0.98 & 12.4 & n.d. \\
\hline KT-13-2_Jan & ON6 & 38.41782 & 142.3326 & 0 & 9.8 & 6.01 & 0.01 & 0.46 & 13.1 & n.d. \\
\hline KK-13-1_Jun & OT4 & 39.33318 & 142.1663 & 0 & 14.6 & $<0.02$ & $<0.01$ & $<0.01$ & 3.00 & 0.87 \\
\hline KK-13-1_Jun & OT4 & 39.33318 & 142.1663 & 23 & 12.7 & 0.12 & 0.03 & 0.02 & 7.03 & 0.61 \\
\hline KK-13-1_Jun & OT4 & 39.33318 & 142.1663 & 42 & 11.7 & 1.53 & 0.62 & 0.18 & 12.9 & 1.56 \\
\hline KK-13-1_Jun & OT5 & 39.3176 & 142.5002 & 0 & 16.4 & 0.04 & 0.07 & 0.02 & 6.59 & n.d. \\
\hline KK-13-1_Jun & OT6 & 39.33345 & 142.8319 & 0 & 15.4 & $<0.02$ & $<0.01$ & $<0.01$ & 3.00 & 0.86 \\
\hline KK-13-1_Jun & ON1 & 38.42158 & 141.4855 & 0 & 15.4 & $<0.02$ & 0.02 & $<0.01$ & 4.22 & 3.83 \\
\hline KK-13-1_Jun & ON4 & 38.41645 & 141.7497 & 0 & 18.5 & $<0.02$ & 0.03 & $<0.01$ & 4.84 & n.d. \\
\hline KK-13-1_Jun & ON5 & 38.41646 & 142.0007 & 0 & 16.8 & $<0.02$ & 0.10 & $<0.01$ & 11.8 & n.d. \\
\hline KK-13-1_Jun & ON5 & 38.41646 & 142.0007 & 25 & 8.9 & 1.69 & 0.06 & 0.28 & 6.74 & n.d. \\
\hline KK-13-1_Jun & ON5 & 38.41646 & 142.0007 & 44 & 9.2 & 5.00 & 0.05 & 0.37 & 13.8 & n.d. \\
\hline KK-13-1_Jun & ON6 & 38.41773 & 142.3338 & 0 & 14.2 & $<0.02$ & 0.17 & $<0.01$ & 18.6 & n.d. \\
\hline KK-13-1_Jun & ON7 & 38.4167 & 142.6679 & 0 & 19.4 & $<0.02$ & 0.06 & $<0.01$ & 7.59 & n.d. \\
\hline KK-13-1_Jun & ON8 & 38.41681 & 143.0004 & 0 & 19.4 & $<0.02$ & 0.03 & $<0.01$ & 5.03 & n.d. \\
\hline KK-13-6_Sep & OT1 & 39.34126 & 141.9318 & 0 & 22.5 & 22.5 & 1.41 & 0.33 & 72.2 & n.d. \\
\hline KK-13-6_Sep & OT4 & 39.33318 & 142.1663 & 0 & 22.8 & 0.30 & $<0.01$ & 0.04 & 9.54 & 0.76 \\
\hline KK-13-6_Sep & OT4 & 39.33318 & 142.1663 & 38 & 20.4 & 1.16 & $<0.01$ & 0.10 & 15.3 & n.d. \\
\hline KK-13-6_Sep & OT4 & 39.33318 & 142.1663 & 72 & 15.5 & 4.32 & 0.10 & 0.32 & 13.9 & n.d. \\
\hline KK-13-6_Sep & OT5 & 39.3176 & 142.5002 & 0 & 19.1 & 0.16 & $<0.01$ & 0.12 & 1.67 & 2.18 \\
\hline KK-13-6_Sep & OT6 & 39.33345 & 142.8319 & 0 & 20.7 & 0.23 & $<0.01$ & 0.05 & 5.35 & 0.75 \\
\hline KK-13-6_Sep & ON4 & 38.41645 & 141.7497 & 0 & 22.3 & 0.04 & $<0.01$ & 0.03 & 1.95 & 0.64 \\
\hline KK-13-6_Sep & ON5 & 38.41646 & 142.0007 & 0 & 20.0 & $<0.02$ & $<0.01$ & 0.08 & 0.36 & 0.77 \\
\hline KK-13-6_Sep & ON6 & 38.41773 & 142.3338 & 0 & 20.3 & 0.03 & $<0.01$ & 0.13 & 0.49 & 0.45 \\
\hline KK-13-6_Sep & ON7 & 38.4167 & 142.6679 & 0 & 23.4 & 0.08 & $<0.01$ & 0.02 & 5.88 & 13.3 \\
\hline KS-14-2_Mar & OT4 & 39.33318 & 142.1663 & 0 & 2.1 & 11.8 & 0.19 & 1.09 & 11.2 & n.d. \\
\hline KS-14-2_Mar & OT4 & 39.33318 & 142.1663 & 14 & 2.1 & 12.6 & 0.30 & 1.14 & 11.7 & n.d. \\
\hline KS-14-2_Mar & OT4 & 39.33318 & 142.1663 & 26 & 2.1 & 13.3 & 0.22 & 1.19 & 11.7 & n.d. \\
\hline KS-14-2_Mar & OT5 & 39.3176 & 142.5002 & 0 & 1.5 & 15.1 & 0.08 & 1.35 & 11.5 & n.d. \\
\hline KS-14-2_Mar & OT6 & 39.33345 & 142.8319 & 0 & 2.4 & 11.3 & 0.11 & 1.13 & 10.3 & n.d. \\
\hline KS-14-2_Mar & ON4 & 38.41645 & 141.7497 & 0 & 7.4 & 10.1 & 0.08 & 0.77 & 13.6 & n.d. \\
\hline KS-14-2_Mar & ON5 & 38.41646 & 142.0007 & 0 & 7.3 & 10.0 & 0.07 & 0.75 & 13.8 & n.d. \\
\hline KS-14-2_Mar & ON5 & 38.41646 & 142.0007 & 25 & 7.3 & 9.98 & 0.02 & 0.74 & 13.7 & n.d. \\
\hline KS-14-2_Mar & ON5 & 38.41646 & 142.0007 & 65 & 7.3 & 10.1 & 0.04 & 0.75 & 13.7 & n.d. \\
\hline KS-14-2_Mar & ON6 & 38.41773 & 142.3338 & 0 & 7.6 & 9.88 & 0.04 & 0.74 & 13.7 & n.d. \\
\hline KS-14-2_Mar & ON7 & 38.4167 & 142.6679 & 0 & 8.1 & 9.04 & 0.03 & 0.66 & 14.0 & n.d. \\
\hline
\end{tabular}

n.d $=$ not detected 
KT-12-20_Aug (7-12 Aug 2012) KT-12-27_Oct (15-22 Oct 2012) KT-13-2_Jan (19-25 Jan 2013)
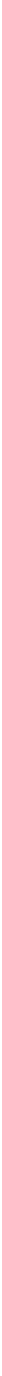

\section{Fig S1 Shiozaki et al.}



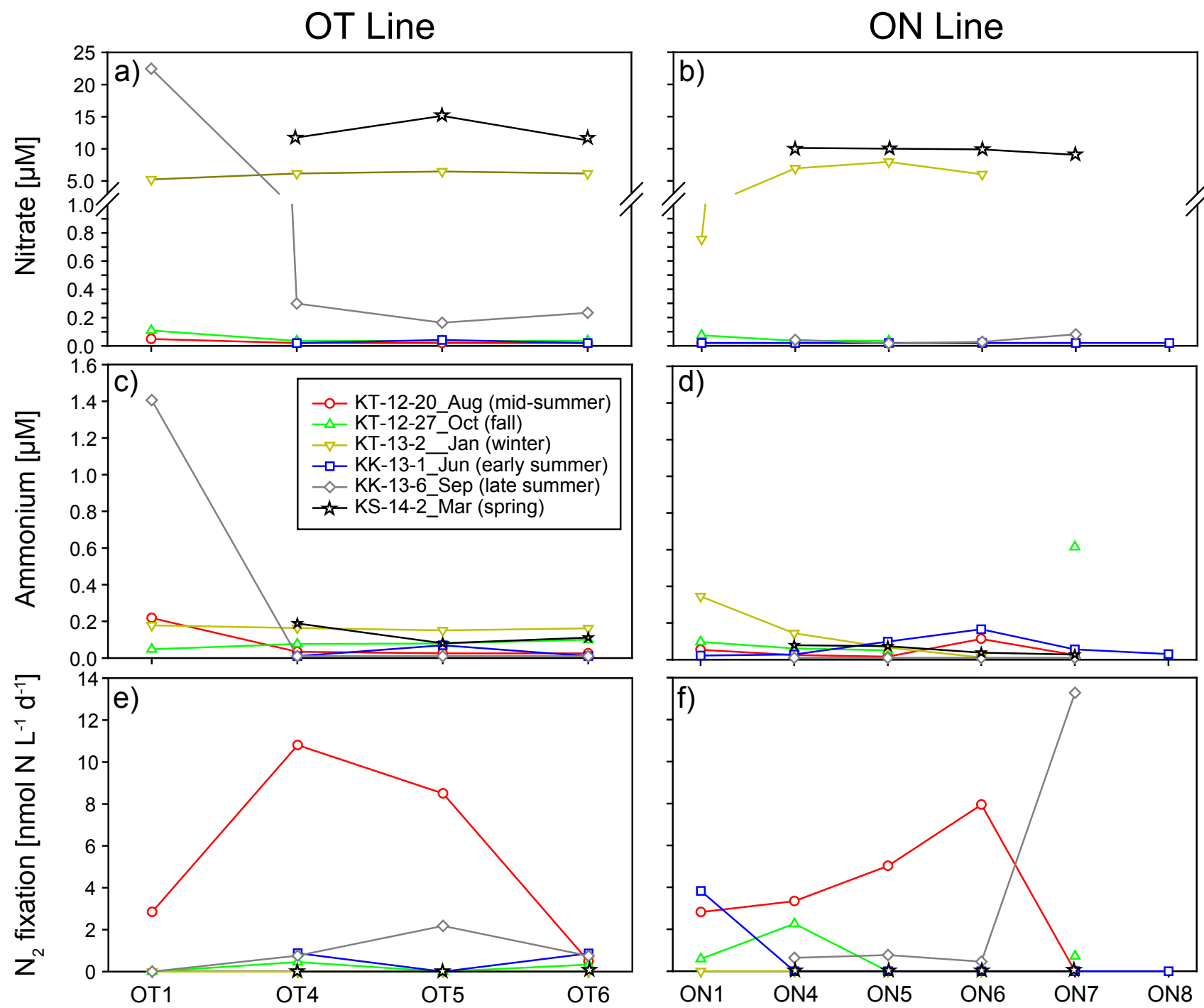

Fig S2 Shiozaki et al. 
O KT-12-20_Aug (mid-summer)

$\triangle$ KT-12-27_Oct (fall)

$\nabla$ KT-13-2_Jan (winter)

$\square$ KK-13-1_Jun (early summer)

$\diamond \mathrm{KK}-13-6 \_$Sep (late summer)

$\star$ KS-14-2_Mar (spring)
65 KT-13-2 ON5-2 ( 7 ON5×3)*

KT-13-2_ON5-14 ( $\nabla$ ON5 $\times 1$ )*

83 AFQ33589 uncultured Bradyr

KK-13-1_OT4-7 (口 OT4X1)*

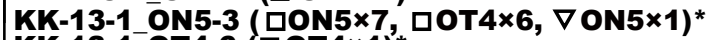

KK-13-1_OT4-6 (口 OT4X1)
PCR contaminant AY225107

-KT-12-20_OT4-10 (OOT4x1)

JX064484 Arabian Sea 09/07/5D

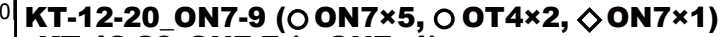

KT-12-20_ON7-7 (O ON7 $\times 1$ )

- AY221812 Xanthobacter flavus

KS-14-2 ON1-12 (†ON1 X1)*

KS-14-2 ON1-16 (†ON1 $\times 1$ )*

53

Q289565 Bradyrhizobium japonicum clone

AB928272 South Indian Ocean Stn.ER9

KS-14-2_ON5-2 (†ON5×4)*

-KS-14-2_ON5-9 ( $九$ ON5 $\times 1$ )*

ACl25908 uncultured soil bacterium

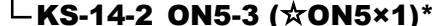

61 KS-14-2_ON1-11 (†ON1 X1)*

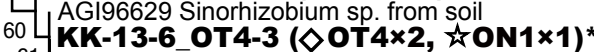

61 - KS-14-2 ON1-3 (

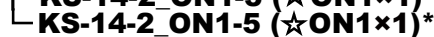

JF429960 Arabian Sea $150 \mathrm{~m}$

BAN45690 Sinorhizobium fredii 96

BAN45690 Sinorhizobiur

72 KT-12-27_ON7-12 ( $\Delta$ ON7 $\times 3)$ *

63

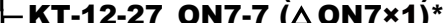

KT-12-27 ON7-10 ( $\Delta$ ON7 ×1)*

AB079632 Rhodobacter blasticus

AB928260 equatorial Indian Ocean Stn.ER8

63 ABX39812 South China Sea Moisander et al 2008

(CAD91359 Dechloromonas sp.

KS-14-2 ON1-6 (*0N1 ×1)

84 JX064480 Arabian Sea 01/08/1E

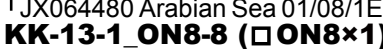

U97116 Azoarcus communis strain SWub3

91 ACP18798 Lake Cadagno chemocline 8.5m

KS-14-2 ON1-10 (« ON1 X1)*

AF378717 Methylomonas methanica strain S1

JX064466 Arabian Sea 10/10/3F

AF059648 Marichromatium purpuratum strain 95Car100a

KT-13-6 OT4-13 (ऽOT4X1)

63 VDU23650 Vibrio diazotrophicus ATCC33466

65 AB928221 Arabian Sea Stn.ER5

M63691 Klebsiella sp. RM1-2

95 ABF61459 Monterey Bay station M1 at 60m

85 KK-13-1 ON1-2 (ON1 X1)

JX064479 Arabian Sea 09/08/4C

AB189453 Pseudomonas azotifigens strain $6 \mathrm{H} 33 \mathrm{~b}$

CAC03734 Pseudomonas stutzeri

87 KT-13-2 OT4-14 ( $\nabla$ OT4×1)

78 ABM67090 sediments of mangrove system

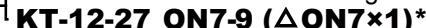

$90-\mathrm{HQ6} 11864$ Eastern North Atlantic 46425A16

HM210403 South Pacific gyre

ABX39738 gamma proteobacteria 24774A11 South China Sea

AB928234 Arabian Sea Stn.ER6

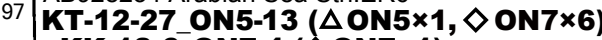

KK-13-6 ON7-4 (>ON7 X1)

AAO67635 Chesapeake Bay Zehr et al 2003

- KS-14-2 ON1-4 († ON1 $\times 1$ )

75 ACl26144 uncultured soil bacterium

98 KT-13-6 OT4-15 (லOT4x1)*

KS-14-2_ON1-15 (†ON1X1)*

ABR01121 Geobacter sp.

70 WP 020677891 Geopsychrobacter electrodiphilus

KT-12-27 ON1-10 ( $\triangle$ ON1 $\times 1)$ *

57 KT-12-27 ON1-14 (AON1X1)*

KK-13-1 ON1-3 (口ON1 ×2)*

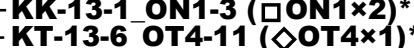

AFD03726 marine sediment mesocosms

KT-12-27 ON1-12 ( $\triangle$ ON1 $\times 2)$

DQ660332 Geoalkalibacter ferrihydriticus

ABA89338 Pelobacter carbinolicus DSM 2380

KK-13-1_ON1-4 (口ON1 ×2)*

ADO25019 mudflat

74 KT-13-2 ON5-6 (7ON5×2)*

54 KS-14-2-ON1-1 (t) 1 ( 12$)$ *

BAN66876 South China Sea Shiozaki et al 2014

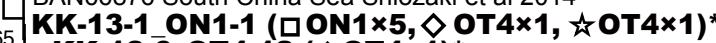

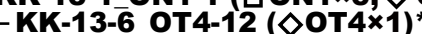
AF296358 Methanosarcina lacustera 
○ KT-12-20_Aug (mid-summer)

$\triangle$ KT-12-27_Oct (fall)

$\nabla$ KT-13-2_Jan (winter)

$\checkmark$ KK-13-1_Jun (early summer)

$\diamond$ KK-13-6_Sep (late summer)

tै KS-14-2_Mar (spring)

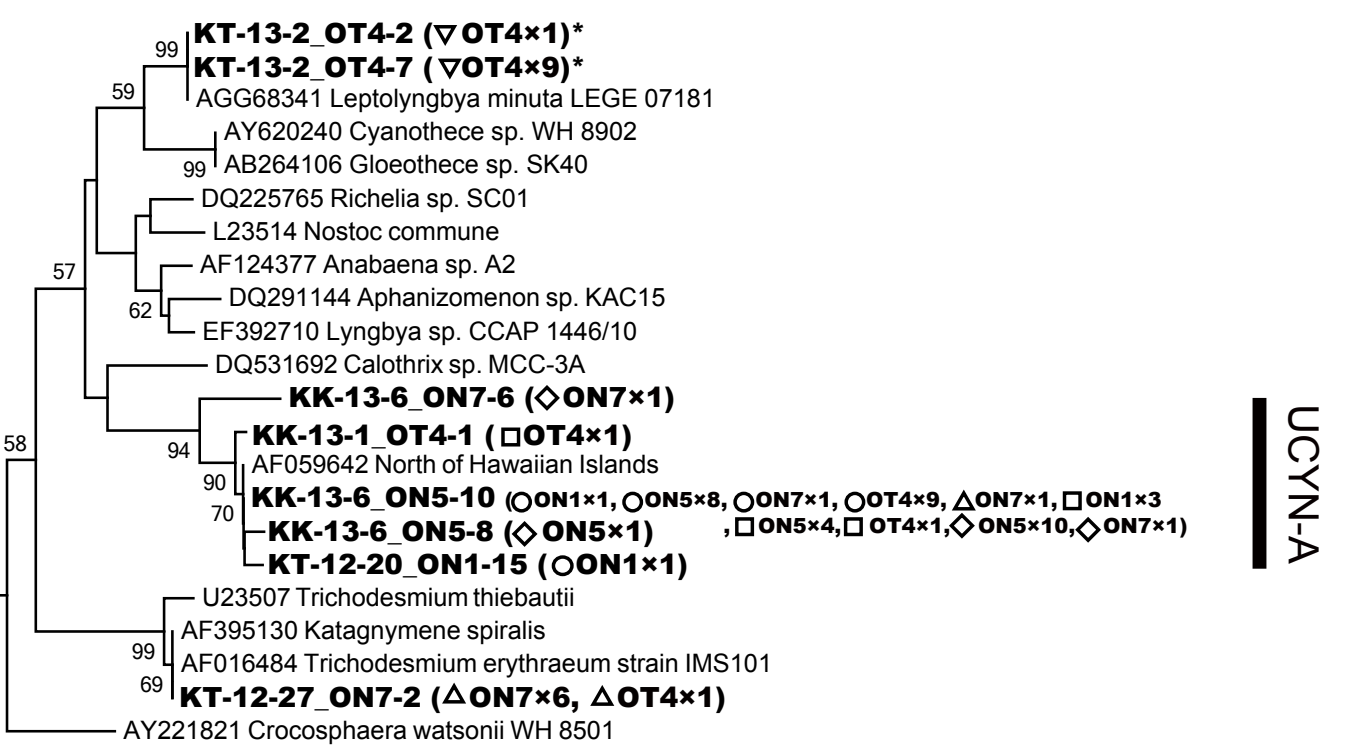

AF296358 Methanosarcina lacustera

\section{Fig S4 Shiozaki et al.}




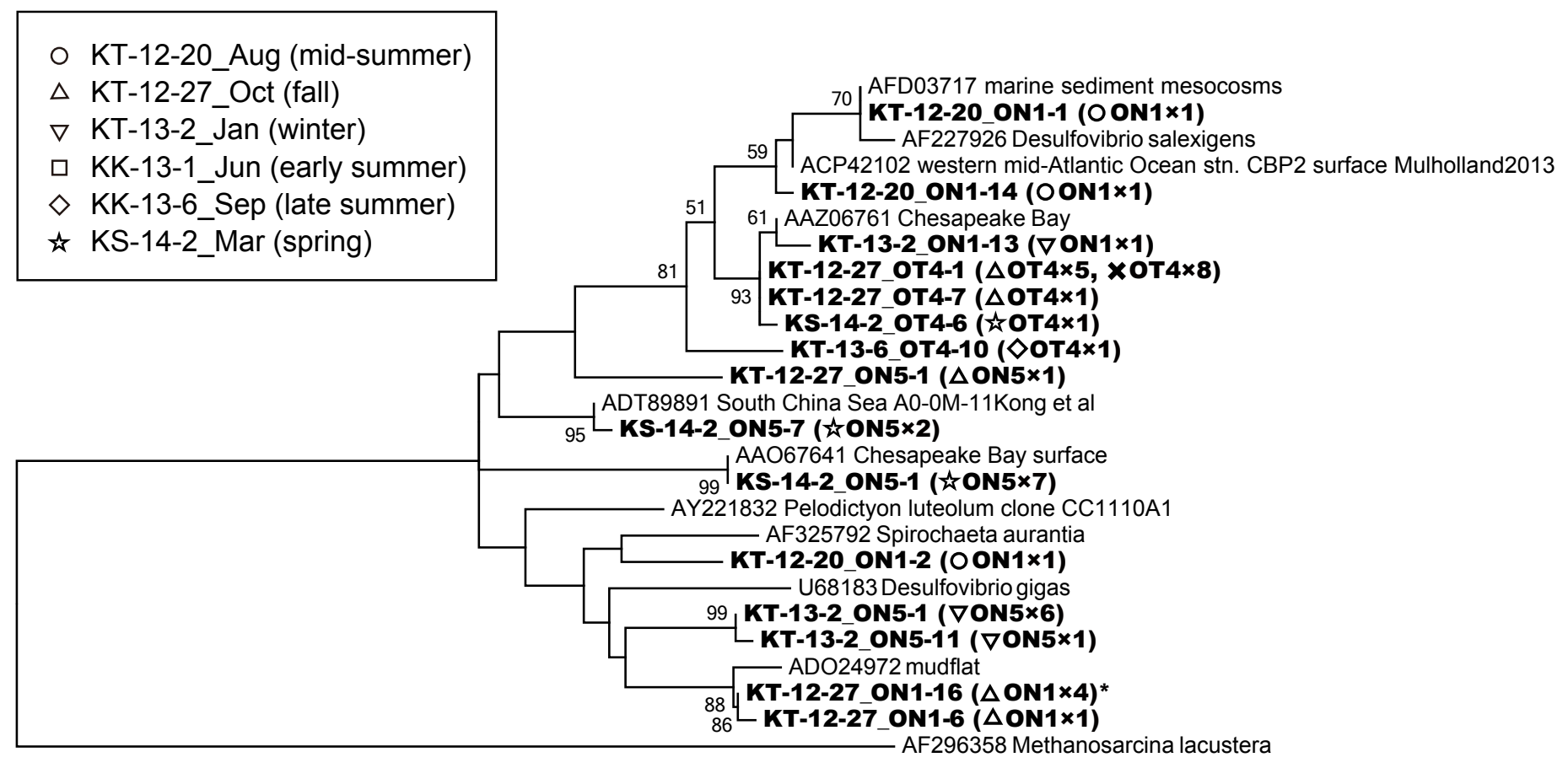



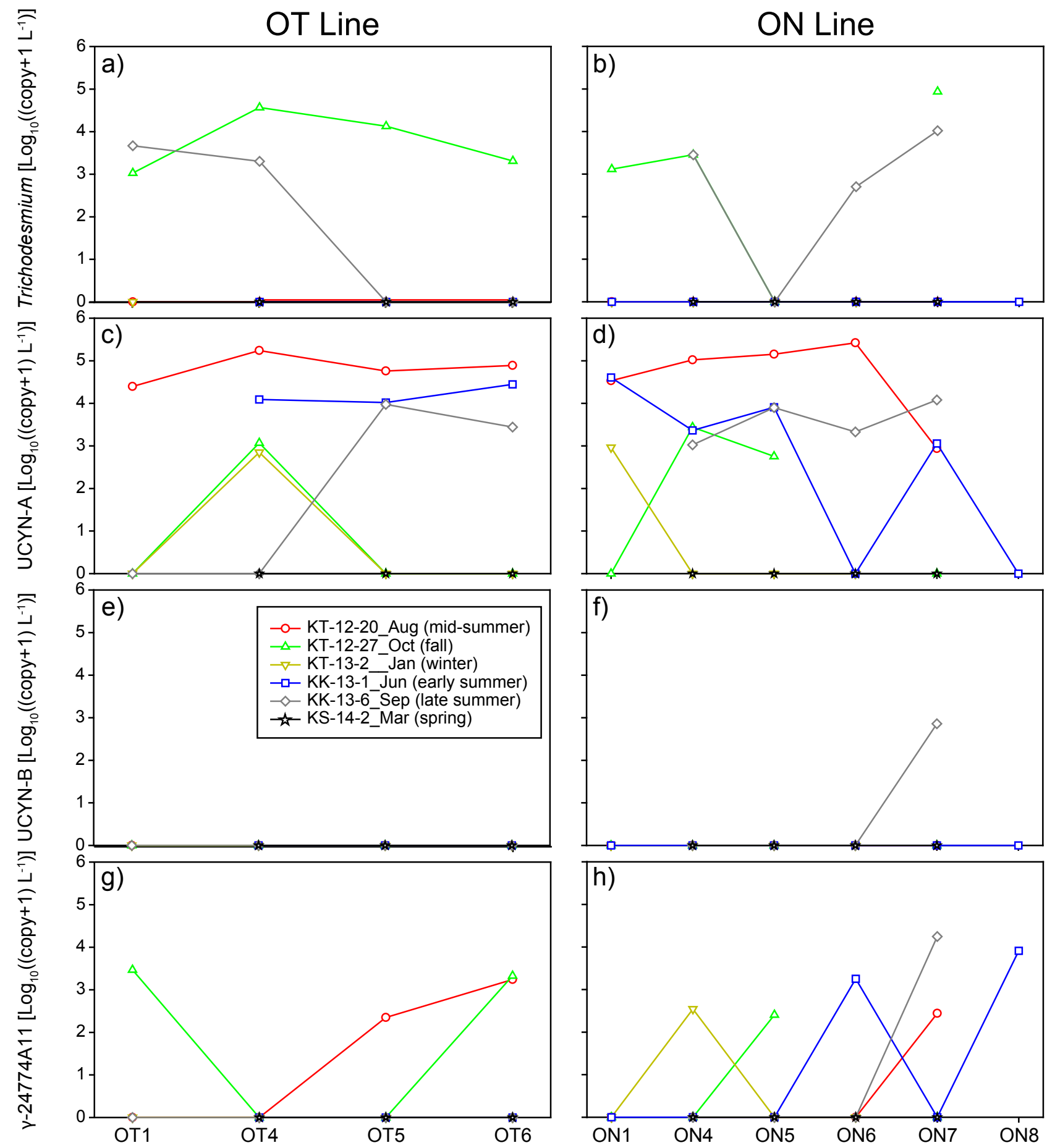

Fig S6 Shiozaki et al. 


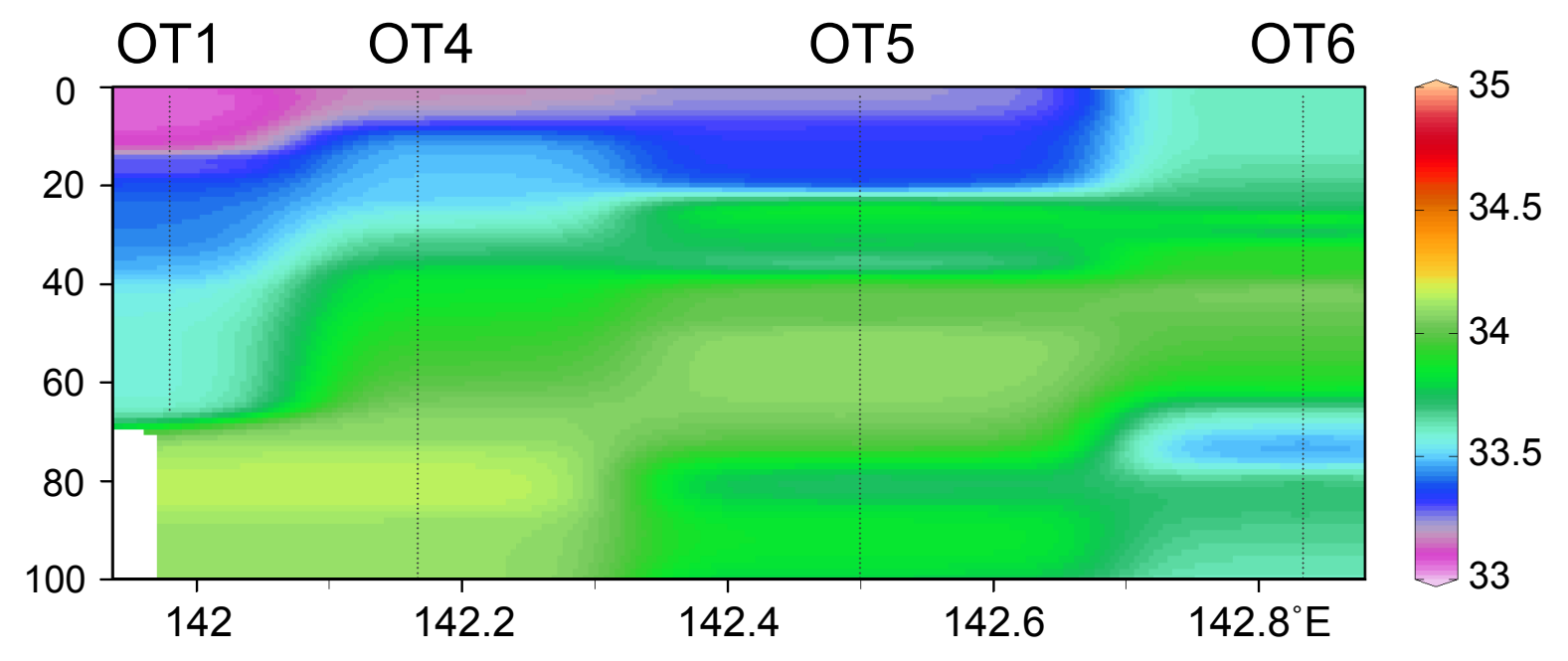

Fig S7 Shiozaki et al. 
KT-12-20_Aug mid-summer

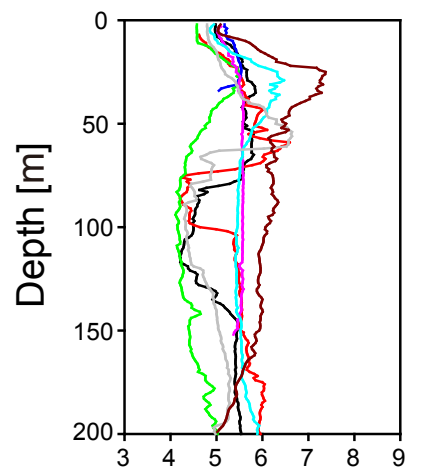

KT-12-27_Oct fall

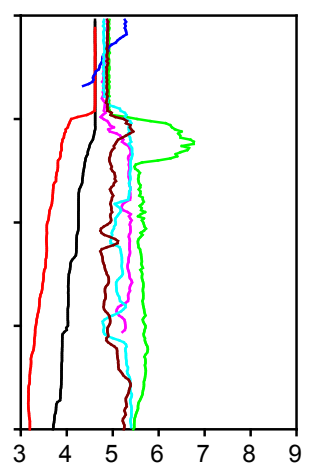

KT-13-2_Jan winter

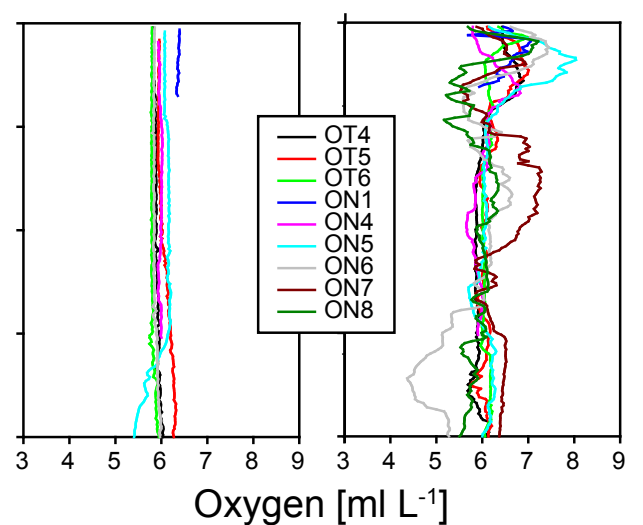

KK-13-6_Sep late summer

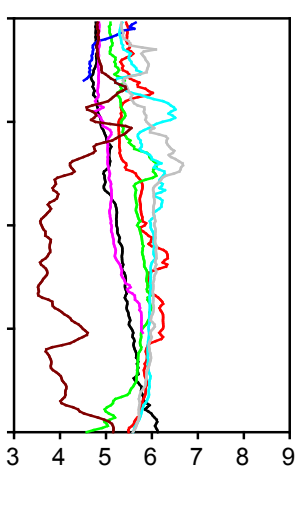

KS-14-2_Mar spring

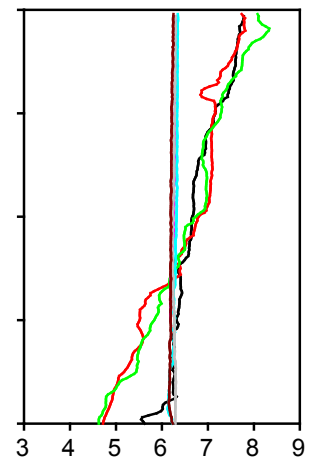

\section{Fig S8 Shiozaki et al.}

